\title{
FOURIER-LIKE KERNELS AS SOLUTIONS OF ODE'S
}

\author{
B.D. AGGARWALA
}

Department of Mathematics and Statistics

The University of Calgary

Calgary, Alberta, Canada, T2N 1N4

(Received July 27, 1993 and in revised form November 30, 1993)

ABSTRACT. In this paper, we generate asymmetric Fourier kernels as solutions of ODE's. These kernels give many previously known kernels as special cases. Several applications are considered.

KEYWORDS AND PHRASES. Fourier kernels, Asymmetric kernels, Anisotropic Plates, Mathematical Biology, Dual Integral Equations.

1991 AMS SUBJECT CLASSIFICATION CODE. 45F10.

\section{INTRODUCTION.}

In a previous paper [1], we indicated how Fourier kernels could be generated as solutions of ordinary differential equations and thus, we generated a large number of hitherto unknown Fourier kernels. In this paper we pursue the same idea and generate some more kernels of a different kind.

2. PRELIMINARIES.

In [1], we noted that solutions of the equation

$$
\frac{\mathrm{d}^{4} \mathrm{u}}{\mathrm{dx}}=\lambda^{4} \mathrm{u}, \quad 0<\mathrm{x}<\infty
$$

which solutions are bounded at infinity, are given by

$$
\mathbf{u}=f_{\cdot} \mathrm{e}^{-\lambda \mathbf{x}}+\mathrm{B} \sin \lambda \mathbf{x}+\mathrm{C} \cos \lambda \mathbf{x} \text {. }
$$

If we now look at the operator $\frac{\mathrm{d}^{4}}{\mathrm{dx}^{4}}$, and notice that



(where' denotes differentiation w.r.t. $x$ ), then, (disregarding the contribution from $x=\infty$ ), the operator $\frac{d^{4}}{d x^{4}}$ is seen to be symmetric over $[0, \infty)$ provided $u$ (and $v$ ) satisfy one of the following conditions:
(1) $\mathbf{u}=\mathbf{v}=0$
and $\quad \mathrm{u}^{\prime}=\mathrm{v}^{\prime}=0$
at
$\mathbf{x}=0$,
(2) $\mathrm{u}=\mathrm{v}=0$
and $u^{\prime \prime}=v^{\prime \prime}=0 \quad$ at
$\mathbf{x}=0$,
(3) $\mathbf{u}^{\prime}=\mathrm{v}^{\prime}=0$
and $\mathrm{u}^{\prime \prime \prime}=\mathrm{v}^{\prime \prime \prime}=0$ at
$\mathrm{x}=0$,
(4) $\mathbf{u}^{\prime \prime}=\mathrm{v}^{\prime \prime}=0$
and $\mathrm{u}^{\prime \prime \prime}=\mathrm{v}^{\prime \prime \prime}=0$ at
$\mathrm{x}=0$. 
In each one of these cases the corresponding solution of equation (1) is a Fourier kernel. In case (1), e.g. we get

$$
u=\sqrt{\frac{1}{\pi}}\left(e^{-\lambda x}-\cos \lambda x+\sin \lambda x\right)
$$

and, we have the pair

$$
\begin{aligned}
& f(x)=\frac{1}{\sqrt{\pi}} \int_{0}^{\infty} \mathrm{A}(\lambda)\left(e^{-\lambda x}-\cos \lambda x+\sin \lambda x\right) d \lambda \\
\Leftrightarrow \quad & A(\lambda)=\frac{1}{\sqrt{\pi}} \int_{0}^{\infty} f(x)\left(e^{-\lambda x}-\cos \lambda x+\sin \lambda x\right) d x .
\end{aligned}
$$

Similarly, case (4) gives

$$
\begin{aligned}
& f(x)=\frac{1}{\sqrt{\pi}} \int_{0}^{\infty} \mathrm{A}(\lambda)\left(\mathrm{e}^{-\lambda x}+\cos \lambda \mathrm{x}-\sin \lambda \mathrm{x}\right) \mathrm{d} \lambda \\
& \Leftrightarrow \quad \mathrm{A}(\lambda)=\frac{1}{\sqrt{\pi}} \int_{0}^{\infty} \mathrm{f}(\mathrm{x})\left(\mathrm{e}^{-\lambda x}+\cos \lambda \mathrm{x}-\sin \lambda \mathrm{x}\right) \mathrm{dx} .
\end{aligned}
$$

and similarly for other cases. $\frac{1}{\sqrt{\pi}}$ in equation (5) is a normalizing factor. The kernels in equations (6) and (7) were noted by Guinand [2], though his arguments were quite different.

We notice that the eigenfunction in equation (5) is symmetric in $x$ and $\lambda$. In this paper we consider eigenfunctions which are not symmetric.

3. ASYMMETRIC KERNALS.

We notice from equation (3) that (disregarding the contribution from $x=\infty$ ) the operator is also symmetric if $u$ (and $v$ ) satisfy any one of the following five conditions:
(1) $\mathrm{u}(0)=\mathrm{v}(0)=0$; $\mathrm{u}^{\prime \prime}(0)=\alpha \mathbf{u}^{\prime}(0), \quad \mathbf{v}^{\prime \prime}(0)=\alpha \mathbf{v}^{\prime}(0)$
(2) $\mathbf{u}^{\prime \prime \prime}(0)=\mathbf{v}^{\prime \prime \prime}(0)=0$;
$\mathrm{u}^{\prime \prime}(0)=\alpha \mathrm{u}^{\prime}(0), \quad \mathrm{v}^{\prime \prime}(0)=\alpha \mathrm{v}^{\prime}(0)$
(3) $\mathbf{u}^{\prime}(0)=\mathbf{v}^{\prime}(0)=0$;
$\mathrm{u}^{\prime \prime \prime}(0)=\alpha \mathrm{u}(0), \quad \mathrm{v}^{\prime \prime \prime}(0)=\alpha \mathrm{v}(0)$
(4) $\mathrm{u}^{\prime \prime}(0)=\mathrm{v}^{\prime \prime}(0)=0$;
$\mathrm{u}^{\prime \prime \prime}(0)=\alpha \mathrm{u}(0), \quad \mathrm{v}^{\prime \prime \prime}(0)=\alpha \mathrm{v}(0)$

and

(5) $u^{\prime \prime \prime}(0)=\alpha u(0), \quad v^{\prime \prime \prime}(0)=\alpha v(0), \quad u^{\prime \prime}(0)=\beta u^{\prime}(0), v^{\prime \prime}(0)=\beta v^{\prime}(0)$.

In equations (8), $\alpha$ and $\beta$ are known (real) constants, assumed positive.

We shall show that in each one of the above cases, the corresponding solutions of equation (1), which are bounded at infinity, generate Fourier-like kernels. Specifically, taking the normalization factors into account, we shall show that for suitable functions $f(x)$ and $A(\lambda)$,

$$
\begin{aligned}
& f(x)=\int_{0}^{\infty} A(\lambda) k(\lambda, x) d \lambda \\
\Leftrightarrow \quad A(\lambda) & =\int_{0}^{\infty} f(x) k(\lambda, x) d x
\end{aligned}
$$

where $\mathbf{k}(\lambda, \mathbf{x})$ takes any one of the following values (corresponding respectively to the five cases in equations (8));

(1) $\mathbf{k}_{1}(\lambda, \mathbf{x})=\sqrt{\frac{2}{\pi}} \frac{1}{\left[(2 \lambda / \alpha+1)^{2}+1\right]^{1 / 2}}\left[\mathrm{e}^{-\lambda \mathbf{x}}-\cos \lambda \mathbf{x}+\frac{2 \lambda+\alpha}{\alpha} \sin \lambda \mathbf{x}\right]$ 
(2) $\mathbf{k}_{2}(\lambda, \mathbf{x})=\sqrt{\frac{2}{\pi}} \frac{1}{\left[(\lambda+2 \alpha)^{2}+\lambda^{2}\right]^{1 / 2}}\left[\lambda \mathrm{e}^{-\lambda \mathbf{x}}-\lambda \sin \lambda \mathbf{x}+(\lambda+2 \alpha) \cos \lambda \mathbf{x}\right]$

(3) $\mathbf{k}_{3}(\lambda, \mathbf{x})=\sqrt{\frac{2}{\pi}} \frac{1}{\left[\left(2 \lambda^{3}+\alpha\right)^{2}+\alpha^{2}\right]^{1 / 2}}\left[\alpha e^{-\lambda x}+\alpha \sin \lambda \mathbf{x}-\left(2 \lambda^{3}+\alpha\right) \cos \lambda \mathbf{x}\right]$

(4) $\mathbf{k}_{\mathbf{4}}(\lambda, \mathbf{x})=\sqrt{\frac{2}{\pi}} \frac{1}{\left[\left(\lambda^{3}+2 \alpha\right)^{2}+\lambda^{6}\right]^{1 / 2}}\left[\lambda^{3} \mathrm{e}^{-\lambda \mathbf{x}}-\left(\lambda^{3}+2 \alpha\right) \sin \lambda \mathbf{x}+\lambda^{3} \cos \lambda \mathbf{x}\right]$

and (5) $\quad \mathrm{k}_{5}(\lambda, \mathbf{x})=\sqrt{\frac{2}{\pi}} \frac{1}{\left[\left(\lambda^{4}+2 \lambda \alpha+\alpha \beta\right)^{2}+\left(\lambda^{4}+2 \beta \lambda^{3}+\alpha \beta\right)^{2}\right]^{1 / 2}} \times$

$$
\left[\left(\lambda^{4}-\alpha \beta\right) \mathrm{e}^{-\lambda \mathrm{x}}-\left(\lambda^{4}+2 \lambda \alpha+\alpha \beta\right) \sin \lambda \mathbf{x}+\left(\lambda^{4}+2 \beta \lambda^{3}+\alpha \beta\right) \cos \lambda \mathrm{x}\right]
$$

It may be noted that, if we put $\alpha=0$ in $\mathrm{k}_{2}(\lambda, \mathbf{x})$, we get the kernel in equations

(7). Also, if we let $\alpha \rightarrow \infty$ in $\mathbf{k}_{1}(\lambda, \mathbf{x})$, we get the kernel in equations (6).

It may also be noted that $\mathbf{k}_{1}, \mathbf{k}_{2}, \mathbf{k}_{3}$ and $\mathbf{k}_{4}$ are all special cases of $\mathbf{k}_{5}(\lambda, \mathbf{x})$.

It may also be noted from equation (3) that the right hand side of this equation vanishes if $\mathrm{u}$ and $\mathrm{v}$ satisfy the following conditions:

$$
\mathbf{u}^{\prime \prime \prime}(0)=\beta \mathrm{u}^{\prime \prime}(0), \mathrm{u}^{\prime}(0)=\alpha \mathrm{u}(0), \mathrm{v}^{\prime \prime \prime}(0)=\alpha \mathrm{v}^{\prime \prime}(0) \text { and } \mathrm{v}^{\prime}(0)=\beta \mathrm{v}(0) .
$$

In this case $\mathbf{k}$ is not a self conjugate kernel. However, we get the pair

$$
\begin{array}{rlrl} 
& f(x) & =\int_{0}^{\infty} A(\lambda) k_{6}(\lambda, x) d \lambda \\
\Leftrightarrow \quad & A(\lambda)=\int_{0}^{\infty} f(x) k_{6}^{*}(\lambda, x) d x
\end{array}
$$

where

$$
\begin{aligned}
& \mathbf{k}_{6}(\lambda, \mathbf{x})=\sqrt{\frac{2}{\pi}}\left[\frac{\lambda(\beta-\alpha) \mathrm{e}^{-\lambda \mathbf{x}}+(2 \alpha \beta+\lambda \alpha+\lambda \beta) \sin \lambda \mathbf{x}+(\alpha+\beta+2 \lambda) \lambda \cos \lambda \mathbf{x}}{\left[(2 \alpha \beta+\lambda \alpha+\lambda \beta)^{2}+(\alpha+\beta+2 \lambda)^{2} \lambda^{2}\right]{ }^{1 / 2}}\right] \\
& \text { and } \mathbf{k}_{6}^{*}(\lambda, \mathbf{x})=\sqrt{\frac{2}{\pi}}\left[\frac{\lambda(\alpha-\beta) \mathrm{e}^{-\lambda \mathbf{x}}+(2 \alpha \beta+\lambda \alpha+\lambda \beta) \sin \lambda \mathbf{x}+(\alpha+\beta+2 \lambda) \lambda \cos \lambda \mathbf{x}}{\left[(2 \alpha \beta+\lambda \alpha+\lambda \beta)^{2}+(\alpha+\beta+2 \lambda)^{2} \lambda^{2}\right]{ }^{1 / 2}}\right]
\end{aligned}
$$

It may be noted that if we put $\beta=0$ in $\mathbf{k}_{6}$, we get

$$
\text { and } \quad \begin{aligned}
\mathbf{k}_{6,1}(\lambda, \mathrm{x}) & =\sqrt{\frac{2}{\pi}}\left[\frac{-\alpha \mathrm{e}^{-\lambda \mathrm{x}}+\alpha \sin \lambda \mathbf{x}+(2 \lambda+\alpha) \cos \lambda \mathbf{x}}{\left[\alpha^{2}+(2 \lambda+\alpha)^{2}\right]^{1 / 2}}\right] \\
\quad \mathbf{k}_{6,1}^{*}(\lambda, \mathbf{x}) & =\sqrt{\frac{2}{\pi}}\left[\frac{\alpha \mathrm{e}^{-\lambda \mathrm{x}}+\alpha \sin \lambda \mathbf{x}+(2 \lambda+\alpha) \cos \lambda \mathrm{x}}{\left[\alpha^{2}+(2 \lambda+\alpha)^{2}\right]^{1 / 2}}\right]
\end{aligned}
$$

as a pair of conjugate kernels. If we now divide all through by $\alpha$ and let $\alpha$ go to infinity, we get the known pair [3]

$$
\begin{aligned}
\mathbf{k}_{6,2}(\lambda, \mathbf{x}) & =\sqrt{\frac{1}{\pi}}\left[-\mathrm{e}^{-\lambda x}+\sin \lambda \mathbf{x}+\cos \lambda \mathrm{x}\right] \\
\text { and } \quad \mathbf{k}_{6,2}^{*}(\lambda, \mathrm{x}) & =\sqrt{\frac{1}{\pi}}\left[\mathrm{e}^{-\lambda \mathrm{x}}+\sin \lambda \mathrm{x}+\cos \lambda \mathrm{x}\right] .
\end{aligned}
$$

Also, in equation (17), if we put $\alpha=\beta$, we get another known kernel [4],

$$
\mathbf{k}_{6,3}(\lambda, \mathbf{x})=\sqrt{\frac{2}{\pi}}\left[\frac{\alpha \sin \lambda \mathbf{x}+\lambda \cos \lambda \mathbf{x}}{\sqrt{\alpha^{2}+\lambda^{2}}}\right]
$$


and

$$
\mathbf{k}_{6,3}^{*}(\lambda, \mathbf{x})=\mathbf{k}_{6,3}(\lambda, \mathbf{x}) .
$$

Since the arguments for showing the validity of equations (9) (or equations (16)) are the same in each case, we shall concentrate on the simplest case, namely $k_{1}(\lambda, x)$.

Proof of Equations (9) for $k=k_{1}(\lambda, x)$

We shall first show that

$$
\begin{aligned}
f(x) & =\int_{0}^{\infty} \mathrm{A}(\lambda) \mathrm{k}_{1}(\lambda, x) d \lambda \\
\Rightarrow \quad \mathrm{A}(\lambda) & =\int_{0}^{\infty} \mathrm{f}(\mathrm{x}) \mathrm{k}_{1}(\lambda, \mathrm{x}) \mathrm{dx}
\end{aligned}
$$

We shall assume that $f(x)$ is in $C^{1}[0, \infty)$ and appropriately well-behaved at infinity. Since now the integral (9b) exists, we may only show that

$$
A(\lambda)=\operatorname{Lim}_{s \rightarrow 0^{+}} \int_{0}^{\infty} e^{-s x} f(x) k_{1}(\lambda, x) d x .
$$

Substituting from (9a), we have

$$
\begin{aligned}
& \int_{0}^{\infty} \mathrm{e}^{-\mathrm{sx}}\left[\int_{0}^{\infty} \mathrm{A}(\mu) \mathrm{k}_{1}(\mu, \mathrm{x}) \mathrm{d} \mu\right] \mathrm{k}_{1}(\lambda, \mathbf{x}) \mathrm{dx} \\
& =\int_{0}^{\infty} \mathrm{A}(\mu)\left[\int_{0}^{\infty} \mathrm{e}^{-s \mathrm{x}} \mathrm{k}_{1}(\lambda, \mathbf{x}) \mathrm{k}_{1}(\mu, \mathbf{x}) \mathrm{dx}\right] \mathrm{d} \mu .
\end{aligned}
$$

The change in the order of integration in equation (22) is justified because of the presence of the term $\mathrm{e}^{-\mathrm{sx}}, \mathrm{s}>0$.

We have, putting $\alpha=\frac{1}{\beta_{1}}$ in equation (10),

$$
\begin{aligned}
& \int_{0}^{\infty} \mathrm{e}^{-\mathrm{sx}} \mathrm{k}_{1}(\lambda, \mathrm{x}) \mathrm{k}_{1}(\mu, \mathrm{x}) \mathrm{dx} \\
& =\left[\frac{2}{\pi}\right] \frac{1}{\sqrt{\left(2 \lambda \beta_{1}+1\right)^{2}+1}} \times \frac{1}{\sqrt{\left(2 \mu \beta_{1}+1\right)^{2}+1}} \times \\
& \int_{0}^{\infty} \mathrm{e}^{-\mathrm{sx}\left(\mathrm{e}^{-\lambda \mathrm{x}}-\cos \lambda \mathrm{x}+\left(2 \lambda \beta_{1}+1\right) \sin \lambda \mathrm{x}\right)\left(\mathrm{e} \mu \mathrm{x}-\cos \mu \mathrm{x}+\left(2 \mu \beta_{1}+1\right) \sin \mu \mathrm{x}\right) \mathrm{dx}} \\
& =\left[\frac{2}{\pi}\right] \frac{1}{\sqrt{\left(2 \lambda \beta_{1}+1\right)^{2}+1}} \times \frac{1}{\sqrt{\left(2 \mu \beta_{1}+1\right)^{2}+1}} \times \mathrm{F}(\lambda, \mu, \mathrm{s}) \\
& =\mathrm{G}(\lambda, \mu, \mathrm{s}), \text { say } \\
& \text { where }
\end{aligned}
$$$$
\begin{aligned}
& \mathrm{F}(\lambda, \mu, \mathrm{s})=\frac{1}{\mathrm{~s}+\lambda+\mu}-\frac{\mathrm{s}+\lambda}{(\mathrm{s}+\lambda)^{2}+\mu^{2}}+\left(2 \mu \beta_{1}+1\right) \frac{\mu}{(\mathrm{s}+\lambda)^{2}+\mu^{2}} \\
& -\frac{\mathrm{s}+\mu}{(\mathrm{s}+\mu)^{2}+\lambda^{2}}+\frac{1}{2} \frac{\mathrm{s}}{\mathrm{s}^{2}+(\lambda+\mu)^{2}}+\frac{1}{2} \frac{\mathrm{s}}{\mathrm{s}^{2}+(\lambda-\mu)^{2}} \\
& -\frac{1}{2}\left(2 \mu \beta_{1}+1\right) \frac{\lambda+\mu}{(\lambda+\mu)^{2}+\mathrm{s}^{2}}+\frac{1}{2}\left(2 \mu \beta_{1}+1\right) \frac{\lambda-\mu}{(\lambda-\mu)^{2}+\mathrm{s}^{2}} \\
& +\left(2 \lambda \beta_{1}+1\right) \frac{\lambda}{\lambda^{2}+(\mathrm{s}+\mu)^{2}}-\left(2 \lambda \beta_{1}+1\right) \cdot \frac{1}{2} \cdot \frac{\lambda+\mu}{(\lambda+\mu)^{2}+\mathrm{s}^{2}}-
\end{aligned}
$$ 


$$
\begin{aligned}
& -\left(2 \lambda \beta_{1}+1\right) \cdot \frac{1}{2} \cdot \frac{\lambda-\mu}{(\lambda-\mu)^{2}+\mathrm{s}^{2}}+\frac{1}{2}\left(2 \lambda \beta_{1}+1\right)\left(2 \mu \beta_{1}+1\right) \times \\
& {\left[\frac{\mathrm{s}}{\mathrm{s}^{2}+(\lambda-\mu)^{2}}-\frac{\mathrm{s}}{\mathrm{s}^{2}+(\lambda+\mu)^{2}}\right] .}
\end{aligned}
$$

From equations (23) and (24) we notice that

(1) $\mathrm{G}(\lambda, \mu, \mathrm{s})$ is continuous in $\lambda, \mu$ and $\mathrm{s}$ in $\lambda>0, \mu>0, \mathrm{~s}>0$,

(2) $\operatorname{Lim} \mathrm{G}(\lambda, \mu, \mathrm{s})=0$, if $\lambda \neq \mu$.

(3) $\operatorname{Lim}_{\varepsilon \rightarrow 0^{+}} \operatorname{Lim}_{\mathrm{s} \rightarrow 0^{+}} \int_{\lambda-\varepsilon}^{\lambda+\varepsilon} \mathrm{G}(\lambda, \mu, \mathrm{s}) \mathrm{d} \mu=1$,

(4) $\mathrm{G}(\lambda, \mu, \mathrm{s})>0$ in $(|\lambda-\mu|<\varepsilon) \cap(0<\mathrm{s}<\delta)$ for sufficiently small $\varepsilon$ and sufficiently small $\delta$,

and (6) $\int_{0}^{\infty}|\mathrm{G}(\lambda, \mu, \mathrm{s})| \mathrm{d} \mu$ exists for all $\lambda$ and all $\mathrm{s}>0$.

From all this, it follows that for given $\lambda>0, \mathrm{a}>0, \mathrm{~b}>0$,

(1) $\int_{a}^{b} G(\lambda, \mu, s) d s$ is bounded uniformly in $s$ in $0<s<\delta$,

and (2) $\begin{aligned} \operatorname{Lim}_{\mathrm{s} \rightarrow 0^{+}} \int_{\mathrm{a}}^{\mathrm{b}} \mathrm{G}(\lambda, \mu, \mathrm{s}) \mathrm{d} \mu & =0, & & \lambda \notin[\mathrm{a}, \mathrm{b} \\ & =1, & & \lambda \in(\mathrm{a}, \mathrm{b})\end{aligned}$

This shows that

$$
\operatorname{Lim}_{\mathrm{s} \rightarrow 0^{+}} \mathrm{G}(\lambda, \mu, \mathrm{s})=\delta(\lambda-\mu), \quad \lambda>0, \mu>0
$$

where $\delta$ is the (generalized) Dirac delta function, and we get

$$
\operatorname{Lim}_{\mathrm{s} \rightarrow 0} \int_{0}^{\infty} \mathrm{A}(\mu) \mathrm{G}(\lambda, \mu, \mathrm{s}) \mathrm{d} \mu=\mathrm{A}(\lambda), \quad \lambda>0,
$$

as desired.

In order to show that the converse is true, i.e. $(9 b) \Rightarrow(9 a)$, we need to show that

$$
\int_{0}^{\infty} \mathbf{k}_{1}(\lambda, \mathbf{x}) \mathbf{k}_{1}(\lambda, \xi) \mathrm{d} \lambda=\delta(\mathbf{x}-\xi), \quad \mathbf{x}>0, \xi>0
$$

Alternatively [5], we may show that the Laplace Transform of the left hand side where $\mathrm{x} \rightarrow \mathrm{p}, \quad \xi \rightarrow \mathrm{q}$ is equal to $1 /(\mathrm{p}+\mathrm{q})$. This is easily shown, since the product of

$$
\int_{0}^{\infty} e^{-p x} k_{1}(\lambda, x) d x \text { and } \int_{0}^{\infty} e^{-q \xi} k_{1}(\lambda, \xi) d \xi
$$

is a rational function of $\lambda$. Taking the Laplace Transform of (9c), changing the order of integration, and substituting, we get the integral of a rational function of $\lambda$, from zero to infinity. Integrating, and simplifying on Mathematica, .we easily get the desired result.

The arguments for other kernels are the same.

\section{SOME APPLICATIONS.}

1. These kernels $\mathbf{k}_{1}, \mathrm{k}_{2}, \ldots, \mathrm{k}_{6}$ would arise if we try to solve the problem of vibrations of a semi-infinite beam whose end $(x=0)$ is subject to appropriate conditions. We try to solve, e.g., 


$$
\frac{\partial^{4} \mathrm{u}}{\partial \mathrm{x}^{4}}+\frac{\partial^{2} \mathrm{u}}{\partial \mathrm{t}^{2}}=0 \quad \text { in } \quad 0<\mathrm{x}<\infty, \quad \mathrm{t}>0
$$

with

$$
\begin{array}{llll}
\text { with } & \mathrm{u}(0, \mathrm{t})=0 & \text { in } & \mathrm{t}>0 \\
& \mathrm{u}_{\mathrm{xx}}(0, \mathrm{t})=\alpha \mathrm{u}_{\mathrm{x}}(0, \mathrm{t}) & \text { in } & \mathrm{t}>0 \\
& \mathrm{u}(\mathrm{x}, 0)=\mathrm{f}(\mathrm{x}) & \text { in } & \mathrm{x}>0 \\
\text { and } & \mathrm{u}_{\mathrm{t}}(\mathrm{x}, 0)=\mathrm{g}(\mathrm{x}) & \text { in } & \mathrm{x}>0
\end{array}
$$

where the subscript denotes partial derivative w.r.t. that variable. This problem gives $\mathbf{u}$ as the deflection in the problem of vibrations of an elastic beam whose end $(x=0)$ is elastically supported, so that the deflection $u$ is zero at $x=0$ in $t>0$, and the bending moment at $\mathbf{x}=0$ is proportional to the slope at $\mathbf{x}=0$. Physical considerations here would require $\alpha \geq 0$.

An appropriate representation of $u$ in this case would be

$$
\mathrm{u}(\mathrm{x}, \mathrm{t})=\int_{0}^{\infty} \mathrm{k}_{1}(\lambda, \mathrm{x})\left[\mathrm{A}(\lambda) \cos \lambda^{2} \mathrm{t}+\frac{\mathrm{B}(\lambda)}{\lambda^{2}} \sin \lambda^{2} \mathrm{t}\right] \mathrm{d} \lambda
$$

and we would require

$$
f(x)=\int_{0}^{\infty} A(\lambda) k_{1}(\lambda, x) d \lambda
$$

and

$$
g(x)=\int_{0}^{\infty} B(\lambda) k_{1}(\lambda, x) d \lambda .
$$

These equations are easily inverted with the help of equtions (9) and then, substitution gives $u . k_{1}(x, y)$ is given by equation (10).

2. The equation

$$
\frac{\partial u}{\partial t}=D_{1} \nabla^{2} u-D_{2} \nabla^{4} u+m^{4} u-f_{1}(x, y)
$$

where $u$ denotes the cell density at a point, occurs in Mathematical Biology. The corresponding steady state equation is

$$
D_{1} \nabla^{2} u-D_{2} \nabla^{4} u+m^{4} u=f_{1}(x, y) .
$$

$m$ is a known constant, depending upon the rate at which the cells multiply. $D_{1}$ here accounts for the short range effects in the diffusion process while $D_{2}$ accounts for the long range ones [6]. If these effects are not isotropic, one may encounter a situation in which the short range effects are dominant in the $y$-direction while the long range ones are dominant in the $\mathbf{x}$-direction. In such a case, after re-scaling, we would get the equation

$$
\frac{\partial^{2} u}{\partial y^{2}}-\frac{\partial^{4} u}{\partial x^{4}}+m^{4} u=f(x, y)
$$

We look for solutions of this equation in $0<\mathrm{y}<\mathrm{L} \cap \mathrm{x}>0$, with the following boundary conditions

$$
\begin{array}{llll}
\mathrm{u}=\mathrm{f}_{2}(\mathrm{y}) & \text { on } \mathrm{x}=0 & \text { in } & 0<\mathrm{y}<\mathrm{L} \\
\frac{\partial \mathrm{u}}{\partial \mathrm{x}}=\mathrm{f}_{3}(\mathrm{y}) & \text { on } \mathrm{x}=0 & \text { in } & 0<\mathrm{y}<\mathrm{L} \\
\mathrm{u}=\mathrm{h}(\mathrm{x}) & \text { on } \mathrm{y}=0 & \text { in } & \mathrm{x}>0
\end{array}
$$


and $\quad \frac{\partial u}{\partial y}=0 \quad$ on $y=L \quad$ in $\quad x>0$

and $|\mathrm{u}|$ bounded as $\mathrm{x} \rightarrow \infty$.

To solve this problem, we write

$\mathrm{u}(\mathrm{x}, \mathrm{y})=\frac{1}{\sqrt{\pi}} \int_{0}^{\infty} \overline{\mathrm{u}}(\lambda, \mathrm{y})\left(\mathrm{e}^{-\lambda \mathrm{x}}-\cos \lambda \mathrm{x}+\sin \lambda \mathrm{x}\right) \mathrm{dx}$

and look for $\overline{\mathrm{u}}(\lambda, \mathrm{y})$. We get

$$
\overline{\mathrm{u}}(\lambda, \mathrm{y})=\frac{1}{\sqrt{\pi}} \int_{0}^{\infty} \mathrm{u}(\mathrm{x}, \mathrm{y})\left(\mathrm{e}^{-\lambda \mathrm{x}}-\cos \lambda \mathrm{x}+\sin \lambda \mathrm{x}\right) \mathrm{dx} .
$$

The kernel in equation (33) is the same as in equation (6). We shall call $\overline{\mathbf{u}}(\lambda, y)$ the F-Transform $(x+\lambda)$ of $u(x, y)$.

Taking the F-Transform of equation (30), we get

$$
\begin{aligned}
\frac{\mathrm{d}^{2} \overline{\mathrm{u}}}{\mathrm{dy}^{2}}-\lambda^{4} \overline{\mathrm{u}}+\mathrm{m}^{4} \overline{\mathrm{u}} & =\overline{\mathrm{f}}(\lambda, \mathrm{y})-\frac{2 \lambda^{2}}{\sqrt{\pi}} \mathrm{f}_{3}(\mathrm{y})-\frac{2 \lambda^{3}}{\sqrt{\pi}} \mathrm{f}_{2}(\mathrm{y}) \\
& =\mathrm{g}(\lambda, \mathrm{y}), \text { say }
\end{aligned}
$$

with

$$
\begin{aligned}
& \overline{\mathrm{u}}(\lambda, 0)=\overline{\mathrm{h}}(\lambda) \\
& \frac{\mathrm{d} \overline{\mathrm{u}}}{\mathrm{dy}}=0 \quad \text { on } \quad \mathrm{y}=\mathrm{L}
\end{aligned}
$$

and

$\bar{f}$ and $\bar{h}$ denote $F$-Transforms of $f$ and $h$ respectively. This problem in $\bar{u}(\lambda, y)$ is easily solvable. If $\mathrm{g} \equiv 0$, we get

$$
\begin{aligned}
& \mathrm{u}(\mathrm{x}, \mathrm{y})=\frac{1}{\sqrt{\pi}} \int_{0}^{\mathrm{m}} \overline{\mathrm{h}}(\lambda) \frac{\cos \left[\left(\sqrt{\mathrm{m}^{4}-\lambda^{4}}\right)(\mathrm{L}-\mathrm{y})\right]}{\cos \left[\left(\sqrt{\mathrm{m}^{4}-\lambda^{4}}\right) \mathrm{L}\right]}\left(\mathrm{e}^{-\lambda \mathbf{x}}-\cos \lambda \mathbf{x}+\sin \lambda \mathbf{x}\right) \mathrm{d} \lambda \\
& +\frac{1}{\sqrt{\pi}} \int_{\mathrm{m}}^{\infty} \overline{\mathrm{h}}(\lambda) \frac{\left.\cosh \left[\sqrt{\left(\lambda^{4}-\mathrm{m}^{4}\right.}\right)(\mathrm{L}-\mathrm{y})\right]}{\cosh \left[\left(\sqrt{\lambda^{4}-\mathrm{m}^{4}}\right) \mathrm{L}\right]}\left(\mathrm{e}^{-\lambda \mathbf{x}}-\cos \lambda \mathbf{x}+\sin \lambda \mathrm{x}\right) \mathrm{d} \lambda .
\end{aligned}
$$

while if $\overline{\mathrm{h}}(\lambda)=0, \overline{\mathrm{u}}(\lambda, \mathrm{y})$ is given by

$$
\begin{aligned}
\overline{\mathrm{u}}(\lambda, \mathrm{y})= & -\int_{0}^{\mathrm{y}} \frac{1}{\omega} \mathrm{g}(\lambda, \xi)(\sin (\omega \xi)) \frac{\cos \omega(\mathrm{L}-\mathrm{y})}{\cos \omega \mathrm{L}} \mathrm{d} \xi \\
& -\int_{0}^{\mathrm{L}} \frac{1}{\omega} \mathrm{g}(\lambda, \xi)(\sin (\omega \mathrm{y})) \frac{\cos \omega(\mathrm{L}-\xi)}{\cos \omega \mathrm{L}} \mathrm{d} \xi, \omega^{2}=\mathrm{m}^{4}-\lambda^{4}>0
\end{aligned}
$$

and

$$
\begin{aligned}
\overline{\mathbf{u}}(\lambda, \mathrm{y})= & -\int_{0}^{\mathrm{y}} \frac{1}{\omega} \mathrm{g}(\lambda, \xi)(\sinh \omega \xi) \frac{\cosh \omega(\mathrm{L}-\mathrm{y})}{\cosh \omega \mathrm{L}} \mathrm{d} \xi \\
& -\int_{\mathrm{y}}^{\mathrm{L}} \frac{1}{\omega} \mathrm{g}(\lambda, \xi)(\sinh \omega \mathrm{y}) \frac{\cosh \omega(\mathrm{L}-\xi)}{\cosh \omega \mathrm{L}} \mathrm{d} \xi, \omega^{2}=\lambda^{4}-\mathrm{m}^{4}>0
\end{aligned}
$$

and then $u(x, y)$ is obtained from equation (32).

Equation (36) suggests that we should take $\mathrm{L}<\pi /\left(2 \mathrm{~m}^{2}\right)$.

3. We consider the bending of an anisotropic plate whose deflection $u(x, y)$ is given by

$$
\frac{\partial^{4} u}{\partial x^{4}}+2 b \frac{\partial^{4} u}{\partial x^{2} \partial y^{2}}+\frac{\partial^{4} u}{\partial y^{4}}=f(x, y)
$$


The case $b=0$ is of some importance [7] and we consider this case here. Also we take $f \equiv 0$. If now, $u$ is governed by the following boundary conditions:

$$
\begin{array}{llll}
u=\frac{\partial u}{\partial x}=0 & \text { along } x=0 & \text { in } & y>0 \\
\frac{\partial u}{\partial x}=f(x) / \sqrt{2} & \text { along } y=0 & \text { in } & 0<x<1 \\
\frac{\partial^{2} u}{\partial x^{2}}=0 & \text { along } y=0 & \text { in } & x>1 \\
u=0 & \text { along } y=0 & \text { in } & x>0
\end{array}
$$

and $|\mathrm{u}|$ bounded at infinity,

an appropriate representation for $u$ in this case would be

$$
\mathbf{u}=\frac{1}{\sqrt{\pi}} \int_{0}^{\infty} \frac{\mathrm{A}(\lambda)}{\lambda}\left(\mathrm{e}^{-\lambda \mathrm{y} / \sqrt{2}} \sin \frac{\lambda \mathrm{y}}{\sqrt{2}}\right)\left(\mathrm{e}^{-\lambda \mathrm{x}}-\cos \lambda \mathrm{x}+\sin \lambda \mathbf{x}\right) \mathrm{d} \lambda
$$

where $f(\lambda)$ is given by

$$
\begin{aligned}
& \frac{1}{\sqrt{\pi}} \int_{0}^{\infty} \mathrm{A}(\lambda)\left(\mathrm{e}^{-\lambda \mathbf{x}}-\cos \lambda \mathrm{x}+\sin \lambda \mathrm{x}\right) \mathrm{d} \lambda=\mathrm{f}(\mathrm{x}), \quad 0<\mathrm{x}<1 \\
& \text { and } \frac{1}{\sqrt{\pi}} \int_{0}^{\infty} \lambda \mathrm{A}(\lambda)\left(\mathrm{e}^{-\lambda \mathrm{x}}-\cos \lambda \mathrm{x}+\sin \lambda \mathrm{x}\right) \mathrm{d} \lambda=0, \quad \mathrm{x}>1 .
\end{aligned}
$$

Such dual integral equations were considered in [8]. We look at these equations again and derive an explicit solution.

If we write

$$
\frac{1}{\sqrt{\pi}} \int_{0}^{\infty} \lambda \mathrm{A}(\lambda)\left(\mathrm{e}^{-\lambda \mathrm{x}}-\cos \lambda \mathrm{x}+\sin \lambda \mathrm{x}\right) \mathrm{d} \lambda=\mathrm{g}(\mathrm{x}), \quad 0<\mathrm{x}<1
$$

we get

$$
\lambda \mathrm{A}(\lambda)=\frac{1}{\sqrt{\pi}} \int_{0}^{1} \mathrm{~g}(\xi)\left(\mathrm{e}^{-\lambda \xi}-\cos \lambda \xi+\sin \lambda \xi\right) \mathrm{d} \xi .
$$

To evaluate $g(\xi)$, we substitute from equation (42) into equation (40a), invert the order of integration and evaluate the inner integral. This gives

$$
\int_{0}^{1} \mathrm{~g}(\xi) \ln \left|\frac{\mathrm{x}^{2}+\xi^{2}}{\mathrm{x}^{2}-\xi^{2}}\right| \mathrm{d} \xi=\pi \mathrm{f}(\mathrm{x}), \quad 0<\mathrm{x}<1 .
$$

This equation is easy to solve [9]. If we define the operator $\mathrm{T}$ by

$$
\mathrm{T} \varphi=\int_{0}^{x} \frac{2 \mathrm{t}^{\frac{3}{2}} \varphi(\mathrm{t}) \mathrm{dt}}{\sqrt{\mathrm{x}^{4}-\mathrm{t}^{4}}}, \quad 0<\mathrm{x}<1
$$

and its conjugate by the requirement that the inner product $(\mathrm{T} \varphi, \psi)=\left(\varphi, \mathrm{T}^{*} \psi\right)$, we get

$$
\mathrm{T}^{*} \psi=\int_{x}^{1} \frac{2 \mathrm{x}^{\frac{3}{2}} \psi(\mathrm{t}) \mathrm{dt}}{\sqrt{\mathrm{t}^{4}-\mathrm{x}^{4}}}
$$

It is now easy to check that

$$
\mathrm{T} \mathrm{T}^{*} \mathrm{~g}=\int_{0}^{1} \ln \left|\frac{\mathrm{x}^{2}+\xi^{2}}{\mathrm{x}^{2}-\xi^{2}}\right| \mathrm{g}(\xi) \mathrm{d} \xi
$$


so that equation (43) may be written as a pair of equations

$$
\mathrm{T}^{*} \mathrm{~g}=\varphi \text { and } \mathrm{T} \varphi=\pi \mathrm{f}(\mathrm{x}) .
$$

These equations give

$$
g(\xi)=-\frac{2}{\pi} \frac{d}{d \xi} \int_{\xi}^{1} \frac{t^{\frac{3}{2}} \varphi(t) d t}{\sqrt{t^{4}-\xi^{4}}}
$$

where $\varphi(t)=\frac{1}{t^{3 / 2}} \frac{d}{d t} \int_{0}^{t} \frac{2 x^{3} f(x)}{\sqrt{t^{4}-x^{4}}} d x$.

For the particular case of $\mathrm{f}(\mathrm{x})=1, \quad 0<\mathrm{x}<1$, we get

$$
g(\xi)=\frac{4}{\pi}\left[\frac{\xi^{3}}{\left(1-\xi^{4}\right)+\sqrt{1-\xi^{4}}}+\frac{1}{\xi}\right], \quad 0<\xi<1 .
$$

The singularity at $\xi=0$ in $g(\xi)$ arises, because "normally" $f(0)=0$ and our assumption of $\mathrm{f}(\mathrm{x})=1$ in $0<\mathrm{x}<1$, creates trouble at zero. If $\mathrm{f}(\mathrm{x})=\mathrm{x}^{2}, 0<\mathrm{x}<1$, this trouble disappears, and we get $g(\xi)=\frac{2 \xi^{3}}{\sqrt{1-\xi^{4}}}$. The square root singularity at $\xi=$ 1 is well-known in other cases. It is easy to find $g(\xi)$ for $f(x)=x^{2 n}, n=0,1,2,3, \cdots$. 4. It is to be noted that other pairs of Dual Integral Equations may be solved in a similar manner. If we have

$$
\begin{aligned}
& \qquad \frac{1}{\sqrt{\pi}} \int_{0}^{\infty} \mathrm{A}(\lambda)\left(\mathrm{e}^{-\lambda \mathrm{x}}+\cos \lambda \mathrm{x}+\sin \lambda \mathrm{x}\right) \mathrm{d} \lambda=\mathrm{f}(\mathrm{x}), \quad 0<\mathrm{x}<1 \\
& \text { and } \frac{1}{\sqrt{\pi}} \int_{0}^{\infty} \lambda \mathrm{A}(\lambda)\left(\mathrm{e}^{-\lambda \mathrm{x}}+\cos \lambda \mathrm{x}+\sin \lambda \mathrm{x}\right) \mathrm{d} \lambda=0, \quad \mathrm{x}>1
\end{aligned}
$$

and we write

$$
\frac{1}{\sqrt{\pi}} \int_{0}^{\infty} \lambda \mathrm{A}(\lambda)\left(\mathrm{e}^{-\lambda \mathrm{x}}+\cos \lambda \mathrm{x}+\sin \lambda \mathrm{x}\right) \mathrm{d} \lambda=\mathrm{g}(\mathrm{x}), \quad \mathrm{x}>1
$$

and proceed as for equations (40), we again arrive at

$$
\int_{0}^{1} g(\xi) \log \left|\frac{x^{2}+\xi^{2}}{x^{2}-\xi^{2}}\right| d \xi=\pi f(x)
$$

which is the same as equation (43).

5. It is interesting to note that the following special case of equation (30).

$$
\frac{\partial^{2} \mathrm{u}}{\partial \mathrm{y}^{2}}-\frac{\partial^{4} \mathrm{u}}{\partial \mathrm{x}^{4}}=0, \quad \mathrm{x}>0, \mathrm{y}>0
$$

behaves like an elliptic equation so that only $u$ or $\frac{\partial u}{\partial y}$ (and not $u$ and $\partial u / \partial t$ as in equation (26)), may be prescribed on $x=0$. We may, e.g. consider the following problem:

Find the solution of equation (53a) subject to the following boundary conditions:

$$
\begin{array}{lll}
u(0, y)=0 & \text { in } & y>0 \\
u_{x}(0, y)=0 & \text { in } & y>0 \\
u(x, 0)=f_{1}(x) & \text { in } & 0<x=1
\end{array}
$$




$$
u_{y}(x, o)=-g_{1}(x) \quad \text { in } \quad x>1
$$

and $|u|$ bounded at infinity.

An appropriate representation of $u(x, y)$ in this case would be

$$
\mathrm{u}(\mathrm{x}, \mathrm{y})=\int_{0}^{\infty} \mathrm{A}(\lambda) \mathrm{k}(\lambda, \mathrm{x}) \mathrm{e}^{-\lambda^{2} \mathrm{y}} \mathrm{d} \lambda
$$

where $\mathbf{k}(\lambda, \mathbf{x})$ is given in equation (5). Other boundary conditions on $\mathbf{y}=0$ will give rise to other kernals.

Equations (53d,e) now give rise to the following dual integral equations:

Find $A(\lambda)$ such that

$$
\begin{array}{lcc}
\int_{0}^{\infty} \mathrm{A}(\lambda) \mathrm{k}(\lambda, \mathbf{x}) \mathrm{d} \lambda=\mathrm{f}_{1}(\mathbf{x}) & \text { in } & 0<\mathbf{x}<1 \\
\int_{0}^{\infty} \lambda^{2} \mathrm{~A}(\lambda) \mathrm{k}(\lambda, \mathbf{x}) \mathrm{d} \lambda=\mathrm{g}_{1}(\mathbf{x}) \quad \text { in } & \mathbf{x}>1 .
\end{array}
$$

This is a new set of dual integral equations which have not been considered previously. We propose to consider such dual integral equations subsequently.

ACKNOWLEDGement. The author is grateful to Professor Cyril Nasim of this department for frequent consultations.

\section{REFERENCES}

1. AGGARWALA, B.D. and NASIM C. Solutions of an Ordinary Differential Equation as a Class of Fourier Kernels, Internat. J. Math. and Math. Sci., Vol. 13, No. 2, (1990), 397-404.

2. GUINAND, A.P. A Class of Fourier Kernels, Quart. J. Math., Oxford (2), 1, (1950), 191-3.

3. NASIM, C. and AGGARWALA, B.D. On a Generalization of Hankel Kernel,



4. TRIM, D.W. Applied Partial Differential Equations, PWS-Kent Publishing Company (1990), p. 274 .

5. JONES, D.S. Generalized Functions, McGraw Hill Book Company (1966).

6. MURRAY, J.D. Mathematical Biology, Springer Verlag (1989), p. 244.

7. KRUG, S. and STEIN, P. Influence Surfaces of Orthogonal Anisotropic Plates, Springer-Verlag, (1961).

8. AGGARWALA, B.D. and NASIM, C. On Dual Integral Equations Arising in Problems of Bending of Anisotropic Plates, Internat. J. Math. and Math. Sci., Vol. 15, No. 3, (1992).

9. POTTER, D. and STIRLING, D.S.G. Integral Equations, Cambridge University Press (1990). 




Advances in

Operations Research





\section{The Scientific} World Journal


International Journal of

Mathematics and

Mathematical

Sciences


Journal of

Applied Mathematics


Submit your manuscripts at http://www.hindawi.com


Mathematical Problems in Engineering
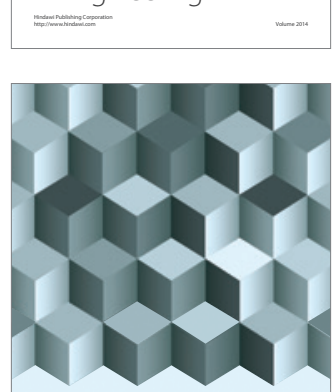

Journal of

Function Spaces




ournal of

Probability and Statistics

Promensencen
